\title{
Effect of Hot-Water Immersion and Foam Rolling on Recovery in Amateur Sepaktakraw Players
}

\author{
Kuswahyudi $^{1, *}$, Firmansyah Dlis ${ }^{1}$, Yasep Setiakarnawijaya ${ }^{1}$, Abdul Gani ${ }^{1}$, Zulham $^{1}$, \\ Ferry Yohannes Wattimena ${ }^{1}$, Bagus Winata ${ }^{2}$ \\ ${ }^{1}$ Faculty of Sport Science, Universitas Negeri Jakarta, Jakarta 13220, Indonesia \\ ${ }^{2}$ Departement of Sport Science, Bandung Institute of Technology, Kota Bandung, Jawa Barat 40132, Indonesia
}

Received November 1, 2020; Revised December 8, 2020; Accepted December 29, 2020

\section{Cite This Paper in the following Citation Styles}

(a): [1] Kuswahyudi, Firmansyah Dlis, Yasep Setiakarnawijaya, Abdul Gani, Zulham, Ferry Yohannes Wattimena, Bagus Winata, "Effect of Hot-Water Immersion and Foam Rolling on Recovery in Amateur Sepaktakraw Players, "International Journal of Human Movement and Sports Sciences, Vol. 8, No. 6, pp. 498 - 504, 2020. DOI: 10.13189/saj.2020.080624.

(b): Kuswahyudi, Firmansyah Dlis, Yasep Setiakarnawijaya, Abdul Gani, Zulham, Ferry Yohannes Wattimena, Bagus Winata (2020). Effect of Hot-Water Immersion and Foam Rolling on Recovery in Amateur Sepaktakraw Players. International Journal of Human Movement and Sports Sciences, 8(6), 498 - 504. DOI: 10.13189/saj.2020.080624.

Copyright $\mathrm{C} 2020$ by authors, all rights reserved. Authors agree that this article remains permanently open access under the terms of the Creative Commons Attribution License 4.0 International License

\begin{abstract}
Sepaktakraw athletes have an extremely high-risk ratio of injury to the lower body, especially the hamstring, when compared to sports like badminton and tennis. This study aims at measuring and comparing the recovery method of the hot-water immersion (HWI) and the foam rolling (FR) in the recovery process of Sepaktakraw athletes. Material and Methods: Overall, 18 Sepaktakraw players aged 18-20 years, who are members of the Sepaktakraw Club of Universitas Negeri Jakarta were involved in this study. 6 participants are randomly assigned to the HWI group, 6 players are assigned to join the FR group, and 6 players are assigned to join the passive recovery group (PR). Three of groups completed measurements pre- and post-recovery for blood lactate concentration and the total quality recovery (TQR). Blood lactate concentration was measured before, immediately, and 15 minutes after recovery. Meanwhile, the total quality recovery (TQR) is taken before the match starts and 15-min after each recovery method was completed. Results: ANOVA showed significant differences between the HWI and PR groups, as well as the FR and PR groups $(p=0.001)$. The post-TQR, ANOVA showed differences between the HWI and PR groups, as well as the FR and PR groups $(\mathrm{p}=$ 0.001). Conclusion: This study showed HWI and FR can reduce blood lactate levels better than the PR group at 15-m after each recovery process in Sepaktakraw athletes. On the other hand, this study shows that the TQR is an accurate predictor index in measuring the effectiveness and
\end{abstract}

quality of a recovery method for Sepaktakraw athletes.

Keywords Vasodilatation, Myofascial Release, Muscle, Training, Blood Lactate

\section{Introduction}

Sepaktakraw began to be competed at the 10th Asian Games in Beijing in 1990, and as a demonstration sport in the 1998 Commonwealth Games in Kuala Lumpur [1]. Since then, Sepaktakraw has begun to expand to various other Asian regions, like China, Japan and India, and it has even been played almost worldwide. Although there is only limited research on Sepaktakraw, several scientific literatures have accurately identified the physiological demands or risk of injury in the Sepaktakraw competition. For instance, Jawis et al., [2] explained that Sepaktakraw is a game of lower work intensity compared to badminton, squash, tennis or basketball. While anaerobic processes supply energy mainly during the explosive movement (e.g. sunback spike, and the other is the roll spike). However, Sepaktakraw players still require aerobic capacity for guarantees continuous energetic support over the entire match and enhance the re-synthesis of phosphocreatine during recovery [2].

Furthermore, in research by Kubo et al., [3] it shows 
Sepaktakraw athletes have an extremely high-risk ratio of injury to the lower body, especially the hamstring, when compared to sports like badminton and tennis. It is because in Sepaktakraw, acrobatic skills and flexibility are required in each process of the movement (e.g. sunback spike, and the other is the roll spike) [2,4]. Apart from these factors, there are several other factors. One of those factors is the exhausting schedule of a Sepaktakraw game in a competition, in which the players have less time to get quality recovery [3]. Those several factors make coaches or other sports practitioners look for a way out to undergo a good recovery for the Sepaktakraw athletes.

Currently, many recovery methods are used to assist athletes in their recovery process [5-7]. For example, the currently popular recovery method, hot-water immersion (HWI), has been observed by several studies to provide benefits to support athletes to reduce muscle soreness, muscle damage, and also improve their performance $[8,9]$. Conversely, there is also some literacy discussing that HWI possesses no beneficial effect as a recovery method for athletes $[10,11]$. On this debate, several experts revealed that different protocols in carrying out HWI therapy (e.g. temperature, time immersion, and body parts immersion) were some reasons that could lead to differences in the results obtained [9].

Foam rolling (FR) is also an alternative method of recovery which is currently repeatedly used by athletes [12]. Apart from being easy and practical to use, FR is believed to provide benefits for athletes in helping their recovery process [13]. It is due to the existence of the effect of myofascial release resulting from the use of FR on the athlete's body, so that it can improve arterial function, improve vascular endothelial function, reduce muscle soreness, and decrease parasympathetic nervous system activity [14]. Although some previous studies have explained and disclosed the benefits of FR $[15,16]$, some previous studies also have shown there is no benefit in using FR as a recovery method for athletes [17].

To the best author's knowledge, there is no research comparing the recovery methods of HWI and FR to support athletes in their recovery process. Peculiarly, there are no studies measuring which recovery methods are suitable for Sepaktakraw athletes. Therefore, this study aims at measuring and comparing the recovery method of the HWI and the FR in the recovery process of Sepaktakraw athletes. In this study, we exclusively addressed the effects of HWI and FR on perception of recovery and blood lactate concentration after one-off Sepaktakraw match in Sepaktakraw players. we hypothesized that the recovery methods of HWI and FR have the same benefit in reducing blood lactate compared to passive recovery.

\section{Materials and Methods}

All things considered, 18 Sepaktakraw players aged
18-20 years, who are members of the Sepaktakraw Club of Universitas Negeri Jakarta were involved in this study. They were selected to be the subjects in this study, through the screening using several inclusion and exclusion criteria. The inclusion criteria are: involving in systematic Sepaktakraw practice at least 15 hours per week, having been in this sport for a minimum of the last 5 years, having a history of participation in national tournaments and never carrying out the recovery methods of HWI and FR before. Concurrently, the exclusion criteria are: having a history of cardiovascular disease, having a history of musculoskeletal injury 3 months prior to the study began, and having an allergy to hot water. Alcohol and caffeine were unaccepted for about 24 hours prior to the experiments.

\subsection{Study Design}

The study used a parallel, 3-group, purposive sampling, pretest-posttest design to determine the effect of HWI, FR dan passive recovery (PR) in Sepaktakraw players. 6 players are assigned to join the HWI group, to be given HWI therapy after the Sepaktakraw match is over; 6 players are assigned to join the FR group, to be given FR therapy after the Sepaktakraw match is over; and 6 players are assigned to join the PR group, as a control group in this study. The players distribution to join the group is done randomly. Blood lactate concentration was measured before, immediately, and 15 minutes after recovery. Meanwhile, the total quality recovery (TQR) is taken before the match starts and 15-min after each recovery method was completed. For this study, substitutions of players were unallowed during the match. All regulations in Sepaktakraw matches follow the rules imposed by the International Sepaktakraw Federation (ISTAF) (http://Sepaktakraw.org/wp-content/uploads/2018/03/Law -of-the-Game-2016-Quad-as-per-Oct-2016-V1.0.pdf). The research design is displayed in Figure 1.

\subsection{Procedure}

After getting an explanation of the working flow of this study in the familiarization session, all participants are required to take a series of anthropometry tests, conducted a week before the experimental session begins. In the anthropometric measurement session, this study used the Omron HBF-375 Karada Scan Body Composition Scale to assess body weight and body fat percentage of all participants. A stadiometer with $0.1-\mathrm{cm}$ readability (Seca 214 Portable Stadiometer, Cardinal Health, Ohio, USA) was applied to measure body height. Body mass index was calculated as the ratio of body mass (kilograms) and body height (meters) squared.

After the anthropometric measurement session was completed, all participants entered the experimental session according to the group that had been previously 
joined randomly. Measurements of blood lactate concentration and TQR were carried out before the start of the match. This study requires all participants to do a 6-min warm-up (static and dynamic movement). 3 matches are run according to the rules and regulations imposed by ISTAF. Specifically, we encouraged coaches to encourage players to promote their abilities to the maximum in the matches played. All matches are conducted professionally, and based on the coach's established strategy. To make the match atmosphere real for this study, a reward was provided to each team winning the match played. Polar RS400 sports watch (Finland) was used to monitored of heart rate (HR) during matches.

After the match has been played, all participants immediately enter into each recovery interventions based on the group that has been previously determined. Blood lactate measurements were carried out immediately after the match ended, and $15-\mathrm{m}$ after the recovery process was complete. Meanwhile, the TQR was carried out together at $15-\mathrm{m}$ after the end of the recovery process. After the end of the last test, the participants were allowed to drink water ad libitum to prevent dehydration. The environmental conditions were kept constant ambient temperature $\left(25-27^{\circ} \mathrm{C}\right)$ and relative humidity $(65-75 \%)$ during matches and recovery interventions.

\subsection{Recovery Interventions}

In the recovery process for the HWI group, all participants immersed themselves in a barrel (diameter $250 \mathrm{~cm} \times$ height $76 \mathrm{~cm}$ ). During the immersion process, they immersed their lower limbs for 10 minutes in water with a temperature of $37^{\circ} \mathrm{C}$. During the immersion process, participants stood (passive immersion). To make the temperature regular, this study re-enters hot water if there is a drop in the water's temperature (the water temperatures were measured with a Testo AG $\mathrm{T} 106$ thermometer). Additionally, during HWI interventions, all participants are required to wear minimal clothes.

The Grid Foam Rooler Kettler $(14$ x $33 \mathrm{~cm})$ was used for performing FR exercises. The applications protocol of FR was based on previous protocols. The FR consisted of five exercises, for quadriceps, hamstrings, adductors, gluteal, and gastrocnemius. One minute for all exercises with $15 \mathrm{~s}$ of the rest was given to all participants. All participants start by placing a foam roller at the most distal portion of the muscle and to place as much of their body mass as tolerable on the foam roller at all times. With a rhythm of 50 beats per minute, all participants were instructed to roll their body mass across the foam roller. For the PR group, the players were instructed to sit on a bench for 10 minutes. The players were instructed to not engage in any other form of recovery procedure (i.e., massage, walking, etc.).

\subsection{Physiological Analyses}

Blood lactate measurement used was a Lactate Pro analyzer (Arkray, Shiga, Japan), by taking $100-\mu l$ samples of fingertip capillary blood from each participant. As for perception of recovery, the TQR method was used according to Kenttä and Hassmén to evaluate the player's general perception of recovery [18]. It is where participants being given a TQR index from a scale of 6 to 20. A scale of 6-7 means lack recovery; 8-9 scale means very poor recovery; a scale of 10-11 means poor recovery; 12-13 means reasonable recovery; 14-15 means good recovery; 16-17 means very good recovery; 18-20 means excellent recovery. In the session of $\mathrm{TQR}$ scale measurement, participants have been given an explanation before starting it, and a familiarization session has been carried out for 1 month before the start of this experimental session.
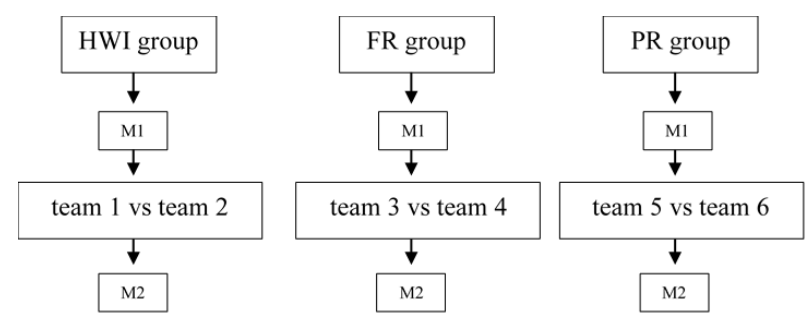

Figure 1. Schematic of matches design based on recovery interventions

Legend: Each group consisted of 3 players; HWI - hot water immersion; FR - foam rolling exercises; PR-passive recovery; M1 - blood lactate and TQR measurements before matches, M2 - blood lactate measurements in immediately after match, and 15 -min after recovery interventions, and TQR measurements in 15-min after 15-min after recovery interventions.

\section{Statistical Analysis}

The values are presented as mean $\pm \mathrm{SD}$. Normal distribution of the sample was checked by using the Shapiro-Wilk test. One-way analysis of variance (ANOVA) with LSD corrected post-hoc analyses was used to calculate the differences between HWI, FR and SJR groups in each physiological parameter. Significant level is accepted if at the level of $p<0.05$. Statistical analysis was performed with the use of the SPSS V.21.0 software.

\section{Results}

Table 1 shows the average anthropometric results of all the participants. Meanwhile, the Table. 2 shows the results of differences of blood lactate concentration and TQR for each group. 
Table 1. Anthropometric characteristics of the three groups of Sepaktakraw players

\begin{tabular}{cccc}
\hline \multirow{2}{*}{ Variable } & \multicolumn{3}{c}{ Groups } \\
\cline { 2 - 4 } & $\begin{array}{c}\text { HWI group } \\
(\overline{\mathrm{x}} \pm \mathrm{SD})\end{array}$ & $\begin{array}{c}\text { FR group } \\
(\overline{\mathrm{x}} \pm \mathrm{SD})\end{array}$ & $\begin{array}{c}\text { SJR group } \\
(\overline{\mathrm{x}} \pm \mathrm{SD})\end{array}$ \\
\hline Age (years) & $18.67 \pm 0.82$ & $18.83 \pm 0.75$ & $18.33 \pm 0.82$ \\
\hline Weight $(\mathrm{kg})$ & $63.53 \pm 4.05$ & $64.42 \pm 2.82$ & $56.80 \pm 3.48$ \\
\hline Height $(\mathrm{cm})$ & $168.25 \pm 3.06$ & $167.95 \pm 3.07$ & $158.43 \pm 2.46$ \\
\hline BMI $\left(\mathrm{kg} / \mathrm{m}^{2}\right)$ & $22.42 \pm 0.68$ & $22.84 \pm 0.75$ & $22.62 \pm 1.03$ \\
\hline
\end{tabular}

\subsection{Blood Lactate Concentration and HR Average}

The results obtained in this study indicate there was no significant difference in the measurement of pre-lactate concentration in the three groups $(p=0.095)$. Furthermore, ANOVA showed there was no significant difference in post-lactate outcomes between the three groups $(p=$ 0.368 ). This result shows the entire group put in the same effort in performing and running the match. It can also be noted in the HR results of each group, showing there was no significant difference between the three groups $(p=$ 0.875). However, the difference between the three groups was merely seen in the blood lactate measurements 15 minutes after the recovery process for each group was complete. In more detail, ANOVA showed significant differences between the HWI and PR groups, as well as the FR and PR groups $(p=0.001)$. It shows there was a significant difference in the reduction of blood lactate, between the group using the recovery methods of HWI and PR group, and the PR group.

\subsection{TQR}

A On the pre-TQR measurement, there were no significant differences between the three groups. It shows that before performing this experimental session, all subjects came with a good recovery condition $(p=0.599)$. However, ANOVA indicated a significant difference in post-TQR. Exclusively, ANOVA showed differences between the HWI and PR groups, as well as the FR and PR groups $(p=0.001)$. When regarded in more detail, the TQR index owned by the HWI group was higher than the FR and PR group. It shows that the effect of HWI leads to better recovery quality when compared to the other two groups.

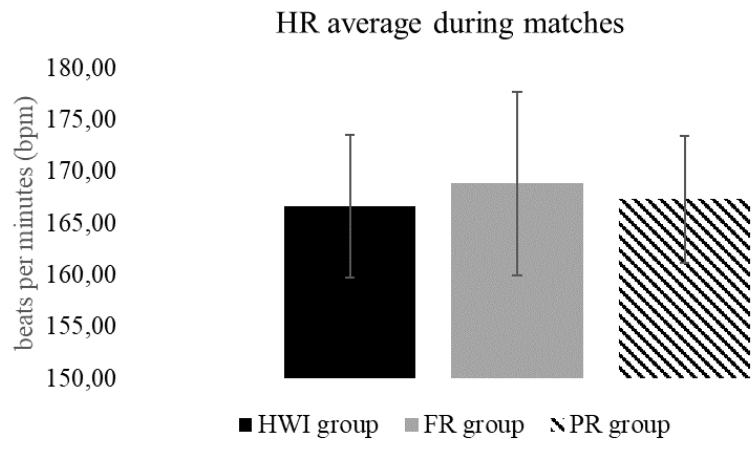

Figure 2. The average of HR in each group

Table 2. Differences in physiological analyzes (blood lactate concentration and TQR) in each group

\begin{tabular}{|c|c|c|c|c|}
\hline \multirow[b]{2}{*}{ Variables } & \multicolumn{3}{|c|}{ Group } & \multirow[t]{2}{*}{$p$-value } \\
\hline & $\begin{array}{c}\text { HWI } \\
(\bar{x} \pm S D)\end{array}$ & $\begin{array}{c}\text { FR } \\
(\bar{x} \pm S D)\end{array}$ & $\begin{array}{c}\text { PR } \\
(\bar{x} \pm S D)\end{array}$ & \\
\hline Pre-test lactate concentration (mmol/l) & $1.13 \pm 0.27$ & $1.12 \pm 0.37$ & $1.13 \pm 0.34$ & 0.995 \\
\hline Post-test lactate concentration (mmol/1) & $7.35 \pm 0.54$ & $6.92 \pm 0.55$ & $7.22 \pm 0.49$ & 0.368 \\
\hline Post 15 -min lactate concentration $(\mathrm{mmol} / \mathrm{l})$ & $2.93 \pm 0.29$ & $3.02 \pm 0.22$ & $4.42 \pm 0.28$ & $0.001 *$ \\
\hline Pre-test TQR & $16.17 \pm 0.75$ & $15.67 \pm 0.82$ & $15.83 \pm 0.98$ & 0.599 \\
\hline Post-test TQR & $15.67 \pm 0.82$ & $14.67 \pm 1.37$ & $10.33 \pm 1.51$ & $0.001 *$ \\
\hline
\end{tabular}

Abbreviations:

HWI - Hot water immersion group; FR - Foam rolling exercise group; PR - Passive recovery group.

The values are presented as mean $\pm \mathrm{SD}$; * values significantly different between the three groups $(\mathrm{p}<0.05)$. 


\section{Discussion}

This study aims at measuring and comparing the recovery methods of HWI and FR for Sepaktakraw athletes. Furthermore, our hypothesis stating the recovery methods of HWI and FR have the same benefit in reducing blood lactate compared to passive recovery. Respect to our hypothesis, this study succeeded in revealing both the recovery methods of HWI and FR have benefits in reducing blood lactate concentration, and they provide good perception of recovery, when compared to the PR group. To the best author's knowledge, this is the first study examining the effect of the recovery method on Sepaktakraw athlete, exactly comparing between HWI and FR on Sepaktakraw athletes.

In this study, it was discovered that a significant decrease in blood lactate occurred in the HWI group compared to the FR and PR groups. Unfortunately, not many previous studies have focused on discussing the effects of HWI on reducing blood lactate. Typically, previous research has always focused on the effects of HWI on the improvement of performance after recovery, the decrease of muscle soreness, and the decrease of creatine kinase $(\mathrm{CK})$ activity in a group of athletes $[8,9]$. Although we are difficult to find supporting data for the research findings, especially to the best author knowledge, there are no studies measuring the effect of HWI as a recovery method in Sepaktakraw athletes, however the previous literacy conducted by Cochrane et al. [19] may explain the phenomenon behind this result. In their study, Cochrane et al., [19] explained that the vasodilatation effect produced by HWI can increase tissue temperature, metabolite production, muscle elasticity, and stimulate blood flow and reduce muscle spasm. Because of this literacy, we argue that an increase in tissue temperature, metabolite production, and stimulation of blood flow in participants after doing HWI can be used as a temporary argument behind the phenomenon of decreasing blood lactate in HWI. Further research is needed involving better physiological measurement methods and parameters to explain this phenomenon.

Although the decrease in blood lactate was more significant in the HWI group compared to the other two groups, the decrease in blood lactate also occurred when comparing the results between the FR group and the PR group. This result is in line with several previous studies finding that the FR method can reduce blood lactate levels $[20,21,22]$. For example, research was conducted by Akinci et al. [20] in which they performed a comparison between FR, active recovery (AR), and neuromuscular electrical stimulation (NMES). In their study, it was explained that the three recovery methods have the ability to reduce blood lactate levels after completing a single bout of circuit-based high-intensity training (CBHIT) at $85 \%$ of the heart rate reserve (HRR). In more detail, the study revealed that the effect of myofascial release caused by FR can reduce edema, muscle soreness, and induce metabolic effects, so that it allows for a decrease in blood lactate in their subjects. Although there are differences in subject characteristics between their study and the current study, this study believe that the effect of myofascial release produced after performing FR exercises was also involved in the Sepaktakraw athletes in this study.

Apart from reporting a decrease in blood lactate levels in the HWI and SJR groups, the current study also demonstrated the validation of the TQR results in line with the decrease in blood lactate in each group. For example, the HWI group experiencing the lowest reduction of blood lactate $(2.93 \pm 0.29)$, followed by the FR group (3.02 \pm 0.22$)$ and the PR group (4.42 \pm 0.28$)$, presented the identical TQR results, in which HWI had better index $(15.67 \pm 0.82)$, followed by the FR group $(14.67 \pm 1.37)$ and the PR group $(10.33 \pm 1.51)$ considered very poor recovery. Needless to say, this study result is in line with the results of previous studies, explaining that TQR is an accurate predictor scale in determining the quality of recovery in athletes of team sports [23].

Although this study proved a positive effect produced by HWI and FR on reducing blood lactate levels, this study also found that many previous studies had shown that these two recovery methods did not produce a satisfactory effect as recovery methods for athletes $[10,11,17]$. Since this study is difficult to find literacy related to the negative effects of HWI on blood lactate, several previous studies have revealed that HWI does not carry out a significant role as a recovery method for athletes in several measurement parameters, such as performance measures, and muscle soreness [10,11]. As one example, Vaile et al. [10] explained that performing HWI for 14 minutes at a temperature of $38{ }^{\circ} \mathrm{C}$ for 5 days does not have an effect on the increase of performance on cycling athletes after they have participated in strenuous training.

Similar to the HWI method, several previous studies also revealed there was no significant effect when using FR as a post exercises recovery method [17,24]. D'amico et al. [24] confirmed that the use of FR as a recovery method for 16 trained males after performing the $800-\mathrm{m}$ treadmill run time did not indicate a decrease in blood lactate levels. On the other hand, many previous studies assumed FR was the same as massage therapy [24,25]. It was possible because of the similar nature of FR to massage therapy, which is "can reducing myofascial trigger points." Unfortunately, even though FR and massage therapy are considered to have similarities, Bielik et al. [26] explained that massage therapy was no better than passive recovery in reducing blood lactate levels at post exercises. This explanation proves that FR and massage may not have a positive effect on reducing blood lactate concentration.

With the existence of the similarities and differences regarding the results obtained in this study, when 
compared with the previous studies findings, this study agree with previous literacy that explained several aspects, like differences in physiological characteristics, numerous types of training levels (junior, senior, amateur or professional), psychological factors, environmental factors, as well as various types of methods or protocols both on HWI (differences in water temperature and immersion duration) and FR (various types of FR material, duration exercises), are some of the factors that can cause differences of results when running or measuring the effectiveness of both recovery methods $[9,27,28]$. We realized this study has several limitations. Firstly, this study did not measure the effectiveness of HWI and FR on performance improvement. Secondly, this study realized that a lack of physiological measurement variables like CK activity, C-reactive protein (CRP), lactate dehydrogenase (LDH), and other physiological measurement parameters was needed to answer an unanswered phenomenon in this study. In addition, future studies are also expected to pay attention to several parameters that can change the final result in the measurement session, like the circadian rhythm, psychological factors and environmental factors. It must be considered to get good results and minimize the effects of bias.

\section{Conclusions}

This study showed HWI and FR can reduce blood lactate levels better than the PR group at $15-\mathrm{m}$ after each recovery process in Sepaktakraw athletes. On the other hand, this study shows that the TQR is an accurate predictor index in measuring the effectiveness and quality of a recovery method for Sepaktakraw athletes. Therefore, we recommend that Sepaktakraw coaches and athletes use the HWI and FR methods as a method of recovery to accelerate post-exercise recovery in athletes, especially for Sepaktakraw players.

\section{Acknowledgment}

The authors would like to thank UNJ Sepak Takraw Club for providing data for the study.

\section{Declaration of Conflicting Interests}

The authors state no conflict of interest with respect to the research, authorship, and/or publication of this article.

\section{Funding}

The authors would like to Research institutions and community service (LPPM UNJ) who funded the project
(LPPM 2019).

\section{REFERENCES}

[1] Aziz A.R., Teo Eikman., Tan B., Chuan T.K, "Sepaktakraw: a descriptive analysis of heart rate and blood lactate response and physiological profile of elite players," Int J Appl Sports Sci, vol 15, pp1-10, 2000.

[2] Jawis M.N., Singh R., Singh H.J., Yassin M.N, "Anthropometric and physiological profiles of sepak takraw players," Br J Sports Med, vol. 39, no. 11, pp. 825-829, 2005. DOI:10.1136/bjsm.2004.016915.

[3] Kubo Y., Nakazato K., Koyama K., Tahara Y., Funaki A., Hiranuma K, "The Relation between Hamstring Strain Injury and Physical Characteristics of Japanese Collegiate Sepak Takraw Players," Int J Sports Med, vol. 37, no. 12, pp. 986-991, 2016. DOI: 10.1055/s-0042-114700.

[4] Leonard Joseph H., Roslizawati N., Safrusahar M.Y., et al, "effect of pubertal developmental stages and lower limb kinetics during vertical jump task in Sepak Takraw sport," Clin Ter, vol. 160, no. 5, pp.403-407, 2009.

[5] Lee E.C., Fragala M.S., Kavouras S.A., Queen R.M., Pryor J.L., Casa DJ, "Biomarkers in Sports and Exercise: Tracking Health, Performance, and Recovery in Athletes," J Strength Cond Res, vol. 31, no. 10, pp. 2920-2937, 2017.

[6] Kellmann M, "Preventing overtraining in athletes in high-intensity sports and stress/recovery monitoring," Scand J Med Sci Sports, vol. 20 Suppl 2, pp. 95-102, 2010.

[7] Haider M.N., Leddy J.J., Pavlesen S., et al, "A systematic review of criteria used to define recovery from sport-related concussion in youth athletes," Br J Sports Med, vol. 52, no. 18, pp. 1179-1190, 2018.

[8] Wilcox C.L., Yanagihara A.A, "Heated Debates: Hot-Water Immersion or Ice Packs as First Aid for Cnidarian Envenomations?," Toxins (Basel), vol. 8, no.4, pp. 97. 2016. DOI: $10.3390 /$ toxins 8040097 .

[9] Versey N.G., Halson S.L., Dawson B.T, "Water immersion recovery for athletes: effect on exercise performance and practical recommendations," Sports Med, vol. 43, no. 11, pp. 1101-1130, 2013. DOI: 10.1007/s40279-013-0063-8

[10] Vaile J., Halson S., Gill N., Dawson B. "Effect of hydrotherapy on recovery from fatigue," Int J Sports Med, vol. 29 , no. 7 , pp. 539-544, 2008. DOI:10.1055/s-2007-989 267.

[11] Kuligowski L.A., Lephart S.M., Giannantonio F.P., Blanc R.O, "Effect of whirlpool therapy on the signs and symptoms of delayed-onset muscle soreness," J Athl Train, vol. 33, no. 3, pp. 222-228, 1998.

[12] Bushell J.E., Dawson S.M., Webster M.M, "Clinical relevance of foam rolling on hip extension angle in a functional lunge position," J Strength Cond Res, vol. 29, no. 9, pp. 2397-2403, 2015. DOI:10.1519/JSC.0000000000000 888 .

[13] Behara B., Jacobson B.H, "Acute effects of deep tissue 
foam rolling and dynamic stretching on muscular strength, power, and flexibility in division I linemen," J Strength Cond Res, vol. 31, no. 4, pp. 888-892, 2017. DOI: $10.1519 /$ JSC.0000000000001051.

[14] Ajimsha M.S., Al-Mudahka N.R., Al-Madzhar J.A, "Effectiveness of myofascial release: systematic review of randomized controlled trials," J Bodyw Mov Ther, vol. 19, no. 1, pp. 102-112, 2015. DOI:10.1016/j.jbmt.2014.06.001.

[15] Wiewelhove T., Döweling A., Schneider C., et al, "A meta-analysis of the effects of foam rolling on performance and recovery," Front Physiol, vol. 10, pp. 376, 2019. DOI. 10.3389/fphys.2019.00376.

[16] Romero-Moraleda B., González-García J., Cuéllar-Rayo Á., Balsalobre-Fernández C., Muñoz-García D., Morencos E, "Effects of vibration and non-vibration foam rolling on recovery after exercise with induced muscle damage," J Sports Sci Med, vol. 18, no. 1, pp.172-180, 2019.

[17] Healey KC., Hatfield D.L., Blanpied P., Dorfman L.R., Riebe D, "The effects of myofascial release with foam rolling on performance," J Strength Cond Res, vol, 28, no.1, pp.61-68, 2014. DOI: 10.1519/JSC.0b013e3182956569.

[18] Kenttä G., Hassmén P, “Overtraining and recovery. A conceptual model," Sports Med, vol. 26, no. 1, pp. 1-16, 1998.

[19] Cochrane D.J, "Alternating hot and cold-water immersion for athlete recovery: a review," Phys Ther Sport, vol. 5, no. 1, pp. 26-32, 2004. DOI: 10.1016/j.ptsp.2003.10.002.

[20] Akinci B., Zenginler Yazgan Y., Altinoluk T, "The effectiveness of three different recovery methods on blood lactate, acute muscle performance, and delayed-onset muscle soreness: a randomized comparative study," J Sports Med Phys Fitness, vol. 60, no. 3, pp. 345-354, 2020. DOI:10.23736/S0022-4707.19.10142-9.

[21] Freiwald, J., Baumgart, C., Kühnemann, M., Hoppe, M.W, "Foam-rolling in sport and therapy - Potential benefits and risks: Part 2 - Positive and adverse effects on athletic performance," Sports Orthop Traumatol, vol. 32, pp.267275, 2016. DOI: 10.1016/j.orthtr.2016.07.002.

[22] Pearcey G.E., Bradbury-Squires D.J., Kawamoto J.E., Drinkwater E.J., Behm D.G., Button D.C, "Foam rolling for delayed-onset muscle soreness and recovery of dynamic performance measures," J Athl Train. Vol. 50, no, 1, pp.5-13, 2015. DOI:10.4085/1062-6050-50.1.01.

[23] Rey E., Padrón-Cabo A., Costa P.B., Barcala-Furelos R, "Effects of Foam Rolling as a Recovery Tool in Professional Soccer Players," J Strength Cond Res, vol. 33, no. 8, pp. 2194-2201, 2019. DOI: 1519/JSC.000000000000 2277.

[24] D'Amico A., Paolone V, "The effect of foam rolling on recovery between two eight hundred metre runs," J Hum Kinet, vol. 57, pp. 97-105, 2017.

[25] DeBruyne D.M., Dewhurst M.M., Fischer K.M., Wojtanowski M.S., Durall C, "Self-Mobilization Using a Foam Roller Versus a Roller Massager: Which Is More Effective for Increasing Hamstrings Flexibility?," J Sport Rehabil, vol. 26, no. 1, pp. 94-100, 2017. DOI: 10.1123/jsr.2015-0035

[26] Bielik V.V, "Effect of different recovery modalities on anaerobic power in off-road cyclists," Bio Sport, vol. 27, pp. 59-63, 2010. DOI: 10.5604/20831862.907953.

[27] Hendricks S., Hill H., Hollander S.D., Lombard W., Parker $\mathrm{R}$, "Effects of foam rolling on performance and recovery: A systematic review of the literature to guide practitioners on the use of foam rolling," J Bodyw Mov Ther, vol. 24, no. 2, pp. 151-174, 2020. DOI: 10.1016/j.jbmt.2019.10.019.

[28] Daskalaki K., Pafis G., Gioftsidou A., Beneka A., Bebetsos E., Malliou P, "Investigation of the Effects of Leg Dominance on Cross-Transfer of Flexibility after a Unilateral Treatment with Foam Roller - A Pilot Study" International Journal of Human Movement and Sports Sciences, vol. 8, no. 3, pp: 79-85, 2020. DOI: https://10.13189/saj.2020.080301. 